\title{
Psychische Verletzungen durch organisationale Veränderungen - ein Gestalt-Coaching-Ansatz
}

\author{
Thomas Bachmann
}

Eingegangen: 30. August 2021 / Angenommen: 5. September 2021 / Online publiziert: 28. Oktober 2021 (C) Der/die Autor(en) 2021

Zusammenfassung Kränkungserleben durch organisationale Veränderungen hat oft tiefe Auswirkungen auf das Selbstvertrauen. Eine mögliche Hypothese besteht darin, dass in Organisationen Entscheidungen für Individuen oft irrational und willkürlich erscheinen. Führungskräfte reagieren in dieser Situation oft mit Kontaktabbruch, was als extreme Form der Kontaktregulation angesehen werden kann. Coaching mit gekränkten und frustrierten Führungskräften stellt eine Herausforderung dar: Für den Klienten kann es ein sehr emotionaler und tiefgreifender und für den Coach ein anspruchsvoller und langwieriger Prozess werden, bis sich Erfolge einstellen. In diesem Artikel wird ein Gestalt-Ansatz für den Umgang mit gekränkten Klienten vorgeschlagen, der sich am Kontaktzyklus aus der Gestalttherapie orientiert.

Schlüsselwörter Psychische Verletzung · Change · Gestalt-Coaching

\section{Psychological wounds through organizational change—a Gestalt coaching approach}

\begin{abstract}
Psychological wounds caused by organizational change often have a deep impact on self-confidence. A possible hypothesis is that in organizations decisions often seem irrational and arbitrary to individuals. Leaders in particular often react in this situation by breaking off contact, which can be seen as an extreme form of contact functions. Coaching with wounded and frustrated leaders is a challenge: For the client it can be a very emotional and profound process and for the coach a demanding and lengthy process until success occurs. This article proposes a Gestalt approach for dealing with wounded clients that is oriented on the Cycle of Experience.
\end{abstract}

Keywords Psychological wounds · Change · Gestalt approach

PD Dr. Thomas Bachmann $(\bowtie)$

Humboldt-Universität zu Berlin, Berlin, Deutschland

E-Mail: thomas.bachmann@hu-berlin.de 
Der Klient: Unser Unternehmen befindet sich im größten Veränderungsprozess seiner Geschichte. Nach dem Dieselskandal und im Zuge der globalen Digitalisierung muss sich alles ändern: die Organisationsstrukturen, die Geschäftsprozesse, die Dinge, die wir tun, und die Kompetenzen, die wir brauchen. Vor einem halben Jahr bestand meine Abteilung aus drei Teams mit jeweils etwa 14 Mitarbeitern. Wir waren für den globalen IT-basierten After-Sales-Service zuständig. Und jetzt? Ich werde zum Teamleiter mit nur vier Mitarbeitern degradiert, und unsere Verantwortung ist auf eine kundenunterstützende App geschrumpft, die wir verwalten. Ich bin wütend. Und ich fühle mich von der Geschäftsleitung schlecht behandelt ... und persönlich verletzt. Warum ich und nicht die anderen? Sowohl mein Vater als auch mein Großvater haben hier im Konzern gearbeitet. Es ist ein Teil unserer Familie und ein Teil unseres Lebens. Ich habe all die Jahre so engagiert gearbeitet und viel mehr Zeit in diesem Unternehmen verbracht als woanders. Ich weiß nicht, was ich in Zukunft machen soll, und es fällt mir schwer, mit anderen Managern zusammen zu sein und ihnen in die Augen zu schauen. Ich bin frustriert.

\section{Einleitung}

Die Beziehung zwischen Person und Organisationen ist ein Feld mit enormen Spannungen und Widersprüchen, denn beide agieren nach gegensätzlichen Logiken, brauchen einander und können nicht ohneeinander existieren. Die Organisation brauchen die Personen, um überhaupt $\mathrm{zu}$ entstehen und ihre Aufgabe zu erfüllen, und die Personen brauchen die Organisationen zur Bedürfnisbefriedigung. Luhmann (2006) beschreibt dabei vier wichtige Steuerungsmedien, mit denen Organisationen die Beziehung zu ,ihren“ Personen regulieren. Diese Medien sind Geld (mehr oder weniger gut bezahlt werden), Macht (gestalten können und Status erlangen), Liebe (Anerkennung und Zugehörigkeit erfahren) und Wahrheit (das „Richtige“ tun). Um die mit den Medien verbundene Bedürfnisbefriedigung zu erreichen, sind die Personen bereit, der Organisation ihre Kompetenzen und ihre Motivation zur Verfügung zu stellen. Soweit der Deal.

Demnach treten Menschen Organisationen bei, um ihre physischen, psychischen und sozialen Bedürfnisse zu befriedigen. Dafür sind sie bereit, in eine mehr oder weniger eingegrenzte und definierte Rolle zu schlüpfen, die mit bestimmten Erwartungen an das Verhalten und die Ergebnisse im Einklang mit den Organisationszwecken ausgestattet ist. Um so viel wie möglich von den Kompetenzen und der Motivation der Individuen - bzw. von ihrem ganzen Selbst - zu erhalten, präsentieren sich Organisationen gerne als eine Art Zuhause, eine Familie oder als ein place to be, wo Menschen Sinn, Anerkennung und Orientierung finden können. Ein bekanntes Beispiel ist die Vision des Google-Campus, in dem Beruf und Privatleben vollständig verschmelzen. Auch die New-Work-Bewegung geht in diese Richtung.

Im Laufe der Zeit verbindet sich die Person im Allgemeinen immer mehr mit ihrer Organisation. Sie wird ein Teil davon (Interpenetration der Systeme nennt Luhmann das) und identifiziert sich mehr und mehr. Das Selbst passt sich im Ver- 
lauf der beruflichen Sozialisation an die organisatorischen Interaktionsmuster an. Dieser Prozess, sich zu identifizieren und Teil eines Interaktionsmusters zu werden, gibt den Menschen das Gefühl, die Organisation zu sein, während sie, aus einer entfernteren Perspektive betrachtet, eher Rollenspieler mit einem Korsett von Erwartungen sind und die Aufgabe haben, die vorgegebenen Muster zu reproduzieren. Diese kritische Sicht auf die Beziehung zwischen Person und Organisation ist im Alltag oft nicht präsent. Diese Sicht und mit ihr all die Widersprüche zwischen Person und Organisation werden dann deutlich, wenn es zu größeren organisationalen Veränderungen kommt. Dann wird erwartet, dass sich die Personen im gleichen Maße mitverändern, und wenn das nicht funktioniert, „leider“ umgesetzt, versetzt, abgesetzt oder freigesetzt werden müssen.

Neben den normalen, permanenten Veränderungsprozessen jeder Organisation sind es die grundlegenden bzw. radikalen Veränderungen, z. B. reorganization, turn arounds, mergers \& aquisition, die tief in die Strukturen und Prozesse der Organisation eingreifen. Dies führt zu disruptiven Veränderungen von Aufgaben, Positionen, Führungsverantwortung, Zugehörigkeit, Teamzusammensetzungen oder Arbeitsorten, welche die Personen oftmals hochgradig persönlich betreffen. Nicht selten fühlen sich Menschen durch die Entscheidungen und deren Auswirkungen im Rahmen solcher Veränderungen tief verletzt.

Eine Kränkung in Zusammenhang mit Veränderungsprozessen, oft auch als strukturelle Kränkung bezeichnet, ist daher immer das Ergebnis einer Interaktion zwischen der Person und dem sozialen System, nämlich der Organisation. Es gibt zwei grundlegende Prozesse, die zu tiefen Verletzungen von Gefühlen von Menschen in Organisationen führen können: (1) wenn Menschen (oft Führungskräfte) durch die Veränderung einen Teil ihrer Macht, ihres Gestaltungsspielraum, ihrer Aufgabe und damit auch ihrer Kompetenzen und schließlich auch ihres Ansehens verlieren, (2) wenn Menschen, die sich sehr engagieren, keine Wertschätzung und Anerkennung für ihre Leistungen erfahren. Die erste Art wird eher durch die Organisation ausgelöst, die zweite eher durch die Person. In beiden Fällen fühlen sich Menschen verletzt und neigen dazu, mit Rückzug, Widerstand, Überanpassung oder Entfremdung zu reagieren.

Es gibt noch einen zusätzlichen Faktor, der eine Kränkung im organisatorischen Kontext besonders verletzend macht: In Organisationen gibt keinen „Schuldigen“, auf den man verweisen könnte, wenn einem so etwas zustößt. Denn in den allermeisten Fällen ist es nicht eine einzelne Person, ein Manager oder eine Führungskraft, die entscheidet, ob und wie sich die Organisation verändert oder nicht. Daher richtet sich der Frust organisationaler Kränkungserfahrungen oft an abstrakte Strukturen wie „das Management“ oder „die das oben“. Ein Klient sagte einmal im Coaching: „Da muss sich Siemens mal überlegen, wie sie zu mir stehen!““

Die Entscheidungen in Organisationen sind das Ergebnis komplexer, langfristiger evolutionärer Prozesse vor allem durch Managementpersonen, Projektteams, externe Berater etc., die den Interessen der Stake- und Shareholder der Organisation folgen. Daher bewerten verschiedene Personen in unterschiedlichen Positionen in oder zur Organisation dieselbe Entscheidung je nach ihrer individuellen Perspektive unterschiedlich. Aus diesem Grund sind organisatorische Entscheidungen und deren Zustandekommen selbst für die Entscheider oft nicht nachvollziehbar. Diese 
begrenzte Rationalität (Simon 1991) und das Fehlen von rechenschaftspflichtigen, persönlich ansprechbaren und Verantwortung übernehmenden Entscheidungsträgern begünstigt nicht selten Klatsch, Gerüchte und Verschwörungstheorien über die Veränderung, die als erste Indikatoren für Abkopplung, Rückzug und innere Emigration der Personen angesehen werden können.

\section{Psychische Verletzungen in Organisationen - Ursachen und Formen}

Psychische Verletzungen durch organisationale Veränderungen haben immer einen direkten negativen Einfluss auf das Selbstwertgefühl, das Selbstkonzept und den Selbstwert. Gerade in unserer narzisstisch-selbstverwirklichenden Zeit, in der alle unter dem Druck leben, ihr perfektes und außergewöhnliches Selbst zu entwickeln, ist jede auch kleine Kränkung ein nachhaltiger Schock für das Selbst und seine Werte, oft begleitet von Scham (Haller 2017). Wie jeder weiß, werden diese Prozesse heutzutage durch die Share-and-Like-Dynamik und den Drang zur Selbstdarstellung in sozialen Netzwerken wie Facebook, Instagram und Co. massiv dynamisiert.

Haller (2017) beschreibt die psychische Verwundung des Selbst als einen langsamen Prozess. Gerade in Organisationen werden veränderungsrelevante Entscheidungen oft über einen langen Zeitraum von einer unprofessionellen oder unbeholfenen Kommunikation der Verantwortlichen begleitet. Das bedeutet, dass es oft nicht ein einzelnes Ereignis ist, das die Verletzung verursacht, sondern eine Reihe von negativen Interaktionen, die das Kränkungs-Wahrnehmen und -Erleben nach und nach verstärken. Haller (2017) unterscheidet verschiedene Typen von psychischen Verletzungen:

- Der erste Typ ist die Kränkung. Die Kränkung, die meist aus einer verbalen Interaktion resultiert, wird offiziell als Beleidigung tituliert, die strafrechtlich geahndet werden kann. In archaischen Kulturen wurden Duelle, Blutrache oder Ehrenmorde zur Rehabilitierung der Ehre als angemessener Umgang mit einer solchen Kränkung eingesetzt. Eine Kränkung lässt also das gekränkte Individuum nach Wiedergutmachung streben.

- Der zweite Typ ist Verbitterung. Verbitterung ist das Ergebnis einer unheilbaren psychischen Verletzung, z. B. ein Trauma bzw. anhaltender Kränkungserfahrungen. Das Individuum fühlt sich durch und durch verbittert. Die Symptome sind Wut, negatives Denken, Abwertung anderer, Depression, Schlaflosigkeit, Psychosen usw. Der psychiatrische Begriff ist die posttraumatische Verbitterungsstörung (Posttraumatic embitterment disorder - PTED)

- Die dritte, die schlimmste und tiefste Art von psychischer Verletzung ist die Demütigung, wenn einem Menschen jegliche Würde genommen wurde, z. B. in Extremsituationen wie Krieg, Folter oder Gefängnisaufenthalt.

- Die vierte ist Mobbing, eine systematisierte Form der psychischen Verletzung, vor allem im organisatorischen Kontext. Hier stehen eine oder mehrere Personen im Mittelpunkt des Mobbingverhaltens. Nach Bassman (1992) wird eine Person, die am Arbeitsplatz gemobbt wird, systematisch abgewertet und respektlos behandelt, oft durch Beschimpfungen oder Überforderung. Auch das Privatleben wird mit 
abgewertet bzw. das Mobbing schlägt bis dahin durch. Personen, die Mobbing erfahren, werden oft durch Mikromanagement von Aufgaben und Zeit schikaniert, durch Drohungen und Einschüchterung gegeißelt, am Zugang zu Möglichkeiten gehindert und in ihren Fähigkeiten herabgestuft, um z.B. Personalabbau zu rechtfertigen. Mobbing kann alle drei o. g. Formen von psychischen Verletzungen enthalten bzw. so erlebt werden.

- Eine weitere spezifische Kategorie ist die narzisstische Kränkung, die z.B. in Organisationen oft ungewollt durch strukturelle Veränderungen verursacht werden kann und zu verschiedenen Arten von negativem Verhalten wie Ironie, Sarkasmus, Zynismus, Bitterkeit usw. bis hin zu permanenter Opposition, Illoyalität und Konflikten führen. Kohut (1973) nennt dieses Phänomen die „narzisstische Wut“.

Führungskräfte, vor allem solche aus Organisationen mit einem mehr ,traditionellen“ Führungsverständnis, sind daher besonders „kränkungsanfällig“, denn sie müssen ,ausreichend“ narzisstisch sein, um ihrer Aufgabe gerecht zu werden. Vor dem Team zu stehen, andere zu inspirieren, sich durch Ideen und Visionen mit Menschen zu verbinden, durchsetzungsfähig und voller Überzeugung zu sein, erfordert unter anderem eine gesunde und moderate Ausprägung narzisstischer Züge (Chamorro-Premuzic 2017). Andererseits sind solche Führungskräfte durch organisatorische Entscheidungen oder Veränderungen stärker gefährdet, narzisstische Kränkungen zu erfahren. Je grandioser man ist, desto tiefer kann man eben auch fallen (Hybris und Nemesis).

In Begriffen der Gestalttherapie gesprochen, tritt durch die Kränkung eine Erschütterung bzw. ein Verlust der Ich-Funktion ein und bewirkt damit eine starke Veränderung des Kontaktverhalten des Selbst (Perls et al. 1951). Gleichzeitig wird durch das Kränkungserleben psychische Energie aktiviert, die das Selbst nach Handlung (nach einem Ausgleich, z. B. Wiedergutmachung, Rache, Ersatzhandlung etc.) streben lässt, die sich jedoch meist nicht umsetzen lässt, da es entweder keine konkreten, erreichbaren Personen gibt, an die man sich richten kann, oder bedingt durch die organisationalen Bedingungen die Möglichkeit dazu schlicht nicht vorhanden ist oder beides. Gekränkte Menschen befinden sich also in einem inneren Spannungszustand.

Nimmt man die Kontaktfunktionen der Gestalttherapie zu Hilfe, kann auf Kränkungen folgendermaßen reagiert werden, um die inneren Spannungen zeitweilig zu regulieren, wenn auch nicht wirklich abzubauen: Durch konfluentes Verhalten kann man sich dem Ungemach anpassen, gute Miene zum bösen Spiel machen, eigene verletzte Bedürfnisse leugnen. Diese Reaktion ermöglicht nur einen schwachen Kontakt mit einem geringen Effekt des inneren Spannungsabbaus. Das Selbst gibt auf, um weitere Verletzungen zu vermeiden. Andere neigen zu starken Projektionen über ihre Situation und vielleicht dazu, anzuklagen oder zu beschuldigen, meist nicht auf konstruktive Art und Weise. Die Energie geht also nach außen und sucht sich ein vermeintliches Ziel zur Reduktion der inneren Spannung. Der dritte Ansatz ist die Kontaktvermeidung durch Retroflexion, typischerweise durch Zurückhalten und Hemmen der Energie, Unterdrückung von Emotionen und Verharren im NichtHandeln. Ein vierter Modus schließlich ist im Egoismus zu sehen, der zu einem distanzierten, kritischen und emotionslosen Verhalten führt, das sich dadurch aus- 
zeichnet, dass man sich nicht auf das Neue, d.h. die veränderte Situation einlässt, die angeboten oder gefordert wird. Durch die Abkoppelung vom inneren emotionalen Erleben kann dabei die Wahrnehmung von Spannung reduziert werden.

Diese klassischen Kontaktunterbrechungen, die von den Begründern der Gestalttherapie 1951 beschrieben wurden, werden von der modernen und weiterentwickelten Gestalttherapie als Kontaktfunktionen bzw. Kontaktformen (Wheeler 1993; Bachmann 2019) verstanden. Durch sie werden die Kontaktprozesse des Selbst mit der Umwelt reguliert. Wenn das Selbst jedoch verletzt ist, können die Kontaktfunktionen in einer sehr starken und destruktiven Weise auftreten und haben die Tendenz, zu dauerhaften Teilen der Persönlichkeit zu werden, wenn sich die Situation nicht ändert und das Individuum seine Situation nicht ergebnisträchtig reflektiert und aktiv bearbeitet. Es geht also darum, die inneren Spannungen konstruktiv umzulenken und abzubauen und damit „offene Gestalten“ zu schließen. Dies kann nicht geschehen, wenn das verletzte Selbst keine Reflexion zulässt bzw. erschütternde Einsichten, aufkommende starke Emotionen oder mögliche Entgleisungen vermeiden möchte. Im schlimmsten Fall kann die narzisstische Verletzung die Ursache und das Ergebnis in einem selbstverstärkenden Prozess sein, in dem das Individuum auf das Negative fokussiert ist, begleitet von negativen Gedanken und Emotionen und der Tendenz, sich selbst und andere permanent abzuwerten.

Aus gestaltherapeutischer Sicht versucht das verletzte Ich also, die veränderte Situation zu bewältigen, indem es Strategien einsetzt, um die negativen Auswirkungen auf das Selbst zu kompensieren und einen weiteren (Gesichts-)Verlust zu vermeiden. Die Balance zwischen der Persönlichkeitsfunktion, also dem Selbstbild (die Summe aller individuellen Erfahrungen), und der Es-Funktion (der möglichen Bedürfnisbefriedigung) muss wieder hergestellt werden. Also muss die Ich-Funktion neue Strategien finden, um die durch die Veränderungen entstandenen Spannungen wieder auszugleichen, d.h. die zur Verfügung stehenden Kontaktfunktionen auf eine andere Art und Weise einzusetzen. Es geht also um eine andere Form der Regulation zwischen Kontakt und Widerstand (Wheeler 1993).

\section{Ein Gestalt-Coaching-Ansatz für narzisstisch gekränkte Führungskräfte}

Kontakt ist der grundlegende Prozess des Lebens, und der Kontakt zwischen dem Individuum und der Umwelt findet an der Kontaktgrenze statt. An dieser Grenze wird das Individuum als autonomer Organismus von der Umwelt abgegrenzt und gleichzeitig findet über diese Grenze der Austausch von Energie und Information statt. Die Kontaktgrenze ist die Voraussetzung und das Ergebnis der Existenz und des Wachstums des Organismus durch Kontakt. In der Gestalttherapie ist der Kontakt das Hauptkonzept, und der therapeutische Ansatz besteht darin, die Fähigkeiten des Individuums, in Kontakt zu treten und erfüllende Kontakterfahrungen zu machen, zu verbessern oder zu ermöglichen (Perls et al. 1951). Die Begründer der Gestalttherapie gingen davon aus, dass im Interaktionsprozess mit anderen das entstehende Selbst das Ergebnis seiner Erfahrungen ist, sodass es offensichtlich ist, dass die Qualität unserer Interaktion mit anderen, z. B. die Intensität, die Tiefe, die emotionalen 
und die sozialen Kompetenzen im Hier und Jetzt, von erfüllenden Kontaktprozessen in der Vergangenheit abhängen. Unterbrochene Kontaktprozesse sind in unserer Psyche noch anhängig und verbrauchen psychische Energie (Zeigarnik 1927). Das unerledigte Geschäft tritt in unseren Träumen auf, führt zu Rumifizieren und Gedankenschleifen, verursacht Unruhe und Rastlosigkeit. Eine narzisstische Verletzung oder eine andere persönliche Kränkung kann also als unterbrochener Kontaktprozess gesehen werden, bei dem die Ich-Funktion versucht, den Kontakt mit Hilfe spezifischer Strategien, z. B. Kontaktfunktionen, zu erfüllen, also die Gestalt zu schließen.

Zur Modellierung des Kontaktprozesses wird in der Gestalttherapie sowie in Coaching und Beratung häufig der Kontaktzyklus verwendet. Dieses Modell beschreibt die Stadien des Kontaktprozesses, die von ansteigender und abfallender psychischer Energie vom Anfang bis zum Ende begleitet werden. Die Stadien sind sensation (etwas tritt innerlich oder äußerlich auf und verursacht eine unspezifische Erregung), awareness (Wahrnehmen und Fühlen der körperlichen, psychischen oder sozialen Bedürfnisse, die mit der Erregung verbunden sind, die Kontaktgrenze entsteht), $a c$ tivation (steigende Energie mobilisiert Projektionen möglicher Handlungen zur Erfüllung der Bedürfnisse durch Bewertung von Objekten, von Anderen, Situationen, Ergebnissen bzw. Pro und Contra), action (aktives und beabsichtigtes Zugehen auf das Neue), contact (aktive Assimilation des Neuen), integration (das Neue, z. B. die Erfahrung, wird zu einem Teil des Selbst, der das Selbst verändert) und withdrawal (das Energieniveau sinkt, und die Kontaktgrenze löst sich wieder auf).

Coaching oder Therapie können Klienten helfen, unvollendete Kontaktprozesse abzuschließen und damit wieder Ressourcen für neue, erfüllende Kontakterfahrungen freizusetzen. Um die narzisstischen Verletzungen oder andere Kränkungserfahrungen zu bearbeiten, ist jedoch ein spezifisches Arbeitsbündnis notwendig, vor allem wenn dies im Rahmen von Coaching geschieht. Wird das Coaching von der Organisation bezahlt, ist besonders genau abzuwägen, inwieweit die Coachingbeziehung für ein solches Anliegen tragfähig ist. Hier besteht aufgrund von klientenseitig fehlendem Vertrauen oder Scham die Gefahr, dass man im Coachingprozess nur an der Oberfläche schürft, obwohl der Coach gut arbeitet und der Klient bereitwillig ist. Letztendlich ist der Coach, wenn er von der Organisation bezahlt wird und dadurch zwangsläufig noch Beziehungen zu anderen Personen in der Organisation hat, damit Teil des kränkenden Systems, demgegenüber man sich vielleicht doch nicht gänzlich öffnen möchte. Aus diesem Grund wird ein privater Kontext empfohlen, um Misserfolge zu vermeiden (vgl. Bachmann 2018). Ein möglicher Prozess (u.a. angelehnt an Haller 2017) wird im Folgenden skizziert.

\section{Vorkontakt - Sensation}

- den Klienten ermutigen, sich selbst einzugestehen, dass er gekränkt ist, also eine narzisstische Verletzung erlitten hat,

- deutlich machen, dass das Reden und Durcharbeiten der Kränkungsgeschichte auch zusammen mit einem professionellen Gegenüber unter Einbeziehung der damit verbundenen Emotionen der einzige konstruktive Weg zu deren Überwindung sind,

- den Mut des Klienten würdigen, sich auf den Prozess einzulassen, und deutlich machen, dass das ein langfristiges Projekt werden kann,

- einen Ausblick auf den bevorstehenden Prozess geben. 
2. Awareness

- Kränkungssituationen der Vergangenheit zusammen mit dem Klienten untersuchen (Auslöser, Reaktionen, Gedanken, Emotionen, Copingversuche),

- den Klienten ermutigen, Emotionen zu benennen und auszudrücken,

- Gedanken, Zuschreibungen und Emotionen sortieren (Was ist meine Rolle in diesem Spiel, was ist die Rolle des anderen? Was genau tut mir weh? Was ist persönlich gemeint und was ist in Bezug auf meine Rolle in der Organisation gemeint? Handelt es sich um ein allgemeines oder ein spezifisches Problem? Gab es in der Vergangenheit, insbesondere in meiner Kindheit, vergleichbare Situationen? etc.),

- dem Klienten Feedback geben und helfen, die aktuellen Kontaktfunktionen zu erkennen, die durch die Beleidigung aktiviert wurden.

\section{Activation}

- erarbeiten, wie andere Beteiligte die Kränkungssituation beurteilen,

- Perspektivwechsel, um zu erfahren, wie das Gegenüber die Kränkungssituation sieht,

- den Klienten ermutigen, um Feedback von Schlüsselpersonen zu bitten,

- Ressourcen und Strategien zur Überwindung der Kränkung finden, die auch schon früher in der Vergangenheit in ähnlichen Situationen funktioniert haben,

- Ideen für zukünftige Konstellationen für das berufliche und private Leben andiskutieren.

4. Action

- Listen schreiben: 1. wertvolle Dinge, auf die ich stolz bin („Schaufenster“), 2. wichtige Dinge, die als prägende Erfahrungen zu mir gehören (,Museum“), 3. Dinge, die ich nicht mitnehmen will, die nicht mehr zu mir gehören, Dinge, die ich loswerden will (,Müllhaufen“),

- Dinge loslassen,

- Losgelassenes aus der Ferne betrachten,

- Welche konstruktiven Elemente stecken auch in den Kränkungsbotschaften?

5. Contact

- Rituale zelebrieren: Abschiedsbriefe schreiben, einen letzten Besuch unternehmen, mit Kollegen sprechen, denen man vertraut usw.,

- künstlerische Wege finden, um die Erfahrung des Verwundet-Seins auszudrücken, z.B. Bücher, Gedichte oder Lieder schreiben, zeichnen, malen, gestalten etc.,

- persönliche Projekte starten, die anderen helfen, die ähnliche Erfahrungen gemacht haben, z. B. Selbsthilfegruppen, Vorträge halten etc.,

6. Nachkontakt - Integration

- dem Klienten durch den Integrationsprozess klar machen, dass die Verletzung immer ein Teil des Selbst sein wird,

- helfen, einen wohlwollenden Blick auf die Narben der Verletzungen zu werfen,

- reflektieren der aktuellen Rolle und über Erwartungen und Anforderungen sprechen,

- Entwickelung neuer persönlicher Ziele und einer Zukunftsperspektive,

- über den Sinn des Lebens sprechen. 
Dieser Prozess kann sich über mehrere Sitzungen erstrecken und muss auch nicht linear verlaufen. Es ist sehr wahrscheinlich, dass man im Verlauf des Prozesses an bestimmte Punkte zurückkehrt, von denen man dachte, dass sie ausreichend bearbeitet wurden. Der Verlauf ist also eher als ein spiralförmiger Prozess zu denken.

Der Klient: Ich habe ein Jahr gebraucht, um die Situation auf der Arbeit zu überwinden. Es war schwer für mich, zuzugeben, dass ich mich persönlich verletzt fühlte. Ich sagte mir, wenn ich weiter hart arbeite, werden sie sehen, dass ich der Richtige bin, und sie werden mir eine neue Chance geben. Aber tief im Inneren wusste ich, dass dies eine Illusion war. Es gab nicht so etwas wie ein ,sie', es gab nur kalte und unpersönliche Entscheidungsalgorithmen zur Geschäftsoptimierung.

Also fasste ich den Entschluss, mit Hilfe eines Coachs darüber hinwegzukommen. Ich dokumentierte jede Woche Daten über meine Gefühle, meine Gesundheit, die Schlafqualität, die positive Interaktion mit anderen, die Unterstützung durch meine Familie, meine Erfolge bei der Arbeit und so weiter. Ich nannte diese Indikatoren mein persönliches ,Cockpit‘. Ich analysierte die Situation viele Male, ich reflektierte mein Verhalten, die Fehler, die ich machte, und die guten Dinge, die ich tat. Ich versuchte, eine Vision für die Zukunft zu entwickeln, aber es war nicht leicht für mich, all die Emotionen und Enttäuschungen hinter mir zu lassen.

Jetzt, ein Jahr später, ist mir klar geworden, dass ich das Unternehmen verlassen muss. Ich stehe in Kontakt mit einem Headhunter, der mir hilft, eine neue Stelle in einem anderen Unternehmen zu finden. Das Wichtigste für mich ist, dass ich meine Firma jetzt ohne Groll und Ärger verlassen kann. Ich blicke mit Stolz zurück und mit Neugierde nach vorn.

\section{Fazit}

Der vorgeschlagene Prozess zur Bearbeitung von Kränkungen und anderen psychischen Verletzungen, die durch organisationale Veränderungen verursacht wurden, kann Klienten helfen, diese zu überwinden und damit wieder Freiraum für neue Möglichkeiten der persönlichen Entwicklung zu erlangen. Der Prozess orientiert sich am Kontaktzyklus aus der Gestalttherapie. Im Kern geht es darum, die Bewusstheit des Klienten zu fördern, ihm zu helfen, mit den damit verbundenen Emotionen in Kontakt zu kommen und die Möglichkeiten für zukünftige Veränderungen zu erforschen. Ein wichtiger Aspekt ist dabei die Differenzierung der Kränkungserfahrung, um zu entscheiden, was davon integriert und was zurückgewiesen werden muss, um einen Neubeginn zu ermöglichen.

Funding Open Access funding enabled and organized by Projekt DEAL.

Open Access Dieser Artikel wird unter der Creative Commons Namensnennung 4.0 International Lizenz veröffentlicht, welche die Nutzung, Vervielfältigung, Bearbeitung, Verbreitung und Wiedergabe in jeglichem Medium und Format erlaubt, sofern Sie den/die ursprünglichen Autor(en) und die Quelle ord- 
nungsgemäß nennen, einen Link zur Creative Commons Lizenz beifügen und angeben, ob Änderungen vorgenommen wurden.

Die in diesem Artikel enthaltenen Bilder und sonstiges Drittmaterial unterliegen ebenfalls der genannten Creative Commons Lizenz, sofern sich aus der Abbildungslegende nichts anderes ergibt. Sofern das betreffende Material nicht unter der genannten Creative Commons Lizenz steht und die betreffende Handlung nicht nach gesetzlichen Vorschriften erlaubt ist, ist für die oben aufgeführten Weiterverwendungen des Materials die Einwilligung des jeweiligen Rechteinhabers einzuholen.

Weitere Details zur Lizenz entnehmen Sie bitte der Lizenzinformation auf http://creativecommons.org/ licenses/by/4.0/deed.de.

\section{Literatur}

Bachmann, T. (2018). Failure in coaching. Between craft and the art of creating relationships. In S. Kunert (Hrsg.), Failure management. Wiesbaden: Springer VS.

Bachmann, T. (2019). Formen des Kontakts. Heidelberg: Carl Auer.

Bassman, E.S. (1992). Abuse in the workplace: management remedies and bottom line impact. Westport: Quorum Books.

Chamorro-Premuzic, T. (2017). Could your personality derail your career? Harvard Business Review, 95(5), 138-141.

Haller, R. (2017). Die Macht der Kränkung. Wals bei Salzburg: Ecowin.

Kohut, H. (1973). Narzissmus. Eine Theorie der psychoanalytischen Behandlung narzisstischer Persönlichkeitsstörungen. Frankfurt/M.: Suhrkamp.

Luhmann, N. (2006). Soziale Systeme. Frankfurt/M.: Suhrkamp.

Perls, F., Hefferline, R., \& Goodman, P. (1951). Gestalt therapy-Excitement and growth in human personality. New York: The Julian Press.

Simon, H. A. (1991). Bounded rationality and organizational learning. Organization science, 2(1), 125-134.

Wheeler, G. (1993). Kontakt und Widerstand. Ein neuer Zugang zur Gestaltherapie. Köln: EHP.

Zeigarnik, B.G. (1927). Das Behalten erledigter und unerledigter Handlungen. Inaugural-Dissertation. Universität Berlin. Berlin: Springer.

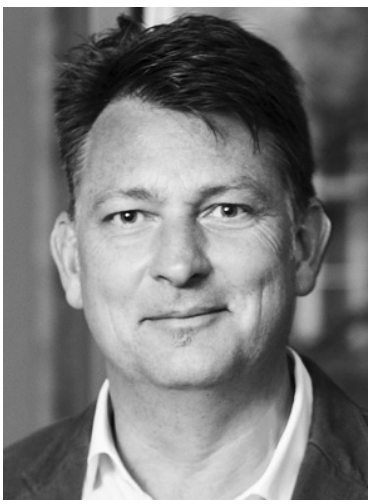

PD Dr. Thomas Bachmann Dipl.-Psych., Dr. rer. nat., Privatdozent, Jahrgang 1964, Mitbegründer und Partner der artop GmbH, Beratungs-, Trainings- und Forschungsinstitut an der Humboldt-Universität zu Berlin. Seit 1993 Berater und Coach für Führungskräfte, Teams und Organisationen. Seit 2001 Coachingausbilder und seit 2004 SeniorCoach im DBVC und Professional Certified Coach der ICF sowie Mitglied der SG. Dozent an der Humboldt-Universität zu Berlin. Forschungsschwerpunkte sind Gruppe, Teams, Agiles Arbeiten und die Professionalisierung von Coaching 